\title{
BNP, Corin and Furin as Potential Biomarkers for Cardiovascular Complications in Type 2 Diabetes Mellitus Patients
}

\author{
Shadia A. Fathy ${ }^{1}$, Fatma F. Abdel Hamid ${ }^{1 *}$, Baker M. Zabut ${ }^{2}$, Amal F. Jamee ${ }^{3}$, Mohamed A. M. Ali ${ }^{1}$ \\ and Ayman M. Abu Mustafa ${ }^{4}$ \\ ${ }^{1}$ Biochemistry Department, Faculty of Science, Ain Shams University, Cairo, Egypt, \\ ${ }^{2}$ Biochemistry Department, Faculty of Science, Islamic University, Gaza, Palestine, \\ ${ }^{3}$ Cardiology Department, El-Shifa Hospital, Gaza, Palestine, \\ ${ }^{4}$ General directorate of human resources development, Ministry of Health, Gaza, Palestine
}

A R T I C L E I N F O

Article history:

Received 27 May 2015

Accepted 07 June 2015

Keywords:

BNP;

Corin;

Furin;

Cardiovascular complications;

Type 2 Diabetes Mellitus.

\begin{abstract}
A B S T R A C T
The number of patients with type 2 diabetes mellitus (T2DM) is progressively increasing, and diabetic cardiovascular complications have become a public health problem. Brain or B-type natriuretic peptide (BNP) is a cardiac hormone synthesized as a pre-pro-peptide. Pro-BNP is produced by cleaving the signal peptide then two proprotein convertases, corin and furin cleave pro-BNP to form a biologically active hormone. Two corin single nucleotide polymorphisms (SNPs) have been reported to alter corin protein conformation and impair its biological activity. We aimed to investigate the potential role of corin and furin in comparison to BNP as biomarkers for predicting cardiovascular complications in T2DM patients. The association of corin gene SNPs with corin levels was also examined. Seventy-five subjects were recruited in this study, including 25 T2DM patients with complications, $25 \mathrm{~T} 2 \mathrm{DM}$ patients without complications as well as 25 healthy subjects. Plasma BNP, corin and furin levels were measured using enzyme-linked immunosorbent assay. Two corin SNPs were genotyped using allele specific oligonucleotide-polymerase chain reaction. Both furin and BNP were found to be more sensitive than corin ( $80 \%$ vs. $56 \%, P=0.008)$, whereas furin showed higher specificity when compared to BNP ( $96 \%$ vs. $84 \%, P=0.041$ ) and corin $(96 \%$ vs. $64 \%, P<0.0001)$ in predicting cardiovascular complications in T2DM patients. Corin SNPs are not associated with corin levels in the study cohort $(P>0.05)$. In conclusion, furin may be useful, either alone or in combination with other biomarkers, for cardiovascular risk stratification assessment in T2DM patients.
\end{abstract}

\section{Introduction}

Type 2 diabetes mellitus (T2DM) has become one of the most important chronic public health problems ${ }^{[1]}$. Cardiovascular diseases (CVDs) are the primary cause of mortality and morbidity in patients with T2DM ${ }^{[2]}$. CVDs are the number one cause of death globally. An estimated 17.3 million people died from CVDs in 2008, representing $30 \%$ of all global deaths. The number of people who die from CVDs will increase to reach 23.3. million by $2030{ }^{[3]}$. CVDs represented the primary cause of Palestinian mortalities reported in 2010, amounting to $25.4 \%$ of the total number of deaths ${ }^{[4]}$.

Atrial natriuretic peptide (ANP) and brain or B-type natriuretic peptide (BNP) are cardiac peptide hormones

* Corresponding author.

E-mail address: fatfarag@ @otmail.com produced primarily by cardiomyocytes of the atrium and ventricle, respectively ${ }^{[5]}$. In response to volume or pressure overload, the expression of ANP and BNP is highly upregulated, increasing their production in the heart and their release into the circulation. The biological effects of ANP and BNP are to promote natriuresis, diuresis, and vasodilation, thereby reducing blood volume and pressure ${ }^{[6]}$. Compared to ANP, BNP has emerged as a superior biomarker for left-ventricular dysfunction and chronic heart failure $(\mathrm{CHF})^{[7]}$. Recently, BNP measurements were found to be the current goldstandard biomarker for the management of $\mathrm{CHF}^{[\mathbf{8}]}$.

The human gene for BNP encodes a 134-amino acid prepro-BNP precursor, which after removal of the amino terminal 26-amino acid signal peptide gives rise to a 108 -amino acid pro-BNP peptide (pro-BNP ${ }_{1-108}$ ). During 
release into circulation, further processing of pro- $\mathrm{BNP}_{1-}$ 108 by a proprotein convertase results in the physiologically active 32-amino acid carboxyl-terminal molecule (BNP-32), derived from amino acids 77 to 108, and an inactive amino-terminal fragment (NT-proBNP), derived from amino acids 1 to $76^{[9]}$.

Proprotein convertases are a family of proteases that cleave target proproteins, generating mature, biologically active polypeptides. Two proprotein convertases, corin ${ }^{[10]}$ and furin ${ }^{[11]}$, are considered the most likely pro-BNP processing enzymes. Corin is a type II transmembrane serine protease ${ }^{[12]}$ that has been identified as the physiological "pro-ANP ${ }^{[13]}$ and proBNP ${ }^{[14]}$ convertase". The enzyme is highly expressed in the heart ${ }^{[10]}$, primarily in cardiomyocytes ${ }^{[15]}$, where it uniquely cleaves the inactive natriuretic peptide precursor molecules into biologically active peptide hormones ${ }^{[16]}$. Furin is a ubiquitously expressed type I transmembrane serine protease involved in the proteolytic processing of a wide range of precursor proteins, including growth factors and their receptors, adhesion molecules, and various metalloproteinases ${ }^{[17]}$. In patients with $\mathrm{HF}$, plasma levels of unprocessed natriuretic peptides are highly elevated ${ }^{[18]}$, suggesting that corin and/or furin activity could not be compensated adequately to meet the demand under the pathological condition ${ }^{[19]}$.

Two non-synonymous and non-conservative single nucleotide polymorphisms (SNPs) have been identified in exon 12 of the human corin gene. The C1757T SNP leads to an amino acid substitution of threonine to isoleucine (T555I), whereas A1796C SNP results in an amino acid substitution of glutamine to proline (Q568P) ${ }^{[20]}$. The T555I and Q568P amino acid substitutions were found to alter corin protein conformation and impair its biological activity by impairing zymogen activation ${ }^{[21]}$. Although there are increasing data to implicate the expression of corin and furin in the pathogenesis of CVDs, the role of corin and furin in the pathophysiology of CVDs remains incompletely understood. In view of the clinical importance of predicting the development of CVDs in patients with T2DM, the present study was designed to compare head to head the diagnostic accuracy of BNP, corin and furin in predicting CVDs in T2DM patients. Furthermore, in an attempt to decrease the uncertainty of the association of corin gene SNPS with plasma corin levels and to provide more conclusive evidence regarding their clinical relevance, we explored the genotype distribution of these SNPs in T2DM patients with or without CVDs as well as in healthy subjects.

\section{Subjects and Methods}

\section{Study design and population}

A total of 50 patients were recruited in this case-control study, including 25 T2DM patients without CVDs, who were referred to the outpatient clinic of the diabetic clinic center, Gaza strip, Palestine and 25 T2DM patients with CVDs, who were referred to the cardio care unit of the Cardiology Department, El-Shifa
Hospital, Gaza strip, Palestine. In addition, 25 healthy subjects, who underwent routine medical check-ups at El-Shifa Hospital and had no medical history of CVDs, were recruited as controls based on normal clinical and laboratory findings. Participants who had conditions that might affect the natriuretic system such as diseases of the liver, chronic kidney disease, pulmonary hypertension, chronic obstructive lung disease, congenital heart disease, critical valvular heart disease, autoimmune diseases, or malignant disease were excluded. The study protocol was approved by the scientific ethical committee of Faculty of Science, Islamic University, Gaza strip, Palestine. A written informed consent was obtained from all the enrolled patients and healthy subjects prior to inclusion into the study in accordance with the Declaration of Helsinki.

\section{Clinical evaluation of the participants}

At the time of study baseline, each participant enrolled in the study was thoroughly surveyed according to a selfadministered questionnaire which was recorded by trained research staff. Participants provided baseline demographics and medical history including gender, age, body mass index (BMI), cigarette smoking status, family history of DM, family history of CVDs, hypertension, asthma, nephropathy, retinopathy, neuropathy, recurrent infections, age at onset of DM, duration of DM, DM medications, age at onset of CVDs, duration of CVDs and CVDs medications.

\section{Clinical diagnosis and definitions}

DM was diagnosed according to criteria set by the American Diabetes Association. DM was defined as the presence of 1 of the following: (1) a fasting plasma glucose of $\geq 126 \mathrm{mg} / \mathrm{dL}$ or (2) a self-reported history of DM and current use of either insulin or an oral hypoglycemic medication ${ }^{[22]}$. The diagnosis of CVDs was performed according to the current guidelines by experienced cardiologists who cared for the patients but were blinded to the study ${ }^{[\mathbf{2 3}, 24]}$.

\section{Biochemical investigations}

Fasting plasma glucose (FPG) level, hemoglobin A1c (HbA1c) level and serum levels of total cholesterol (TC), high-density lipoprotein cholesterol (HDL-C), triacylglycerols (TG), lactate dehydrogenase (LDH), creatine kinase (CK), CK-MB, uric acid (UA) and creatinine $(\mathrm{Cr})$ were measured using BS-300 Chemistry Analyzer (Mindray Bio-Medical Electronics, Shenzhen, China) according to the manufacturer's instructions. Urinary albumin (Alb) level was measured using an immunoturbidimetric assay (Randox Laboratories, Northern Ireland, UK), whereas urinary creatinine level was measured using an enzymatic assay (Randox Laboratories, Northern Ireland, UK) to calculate the urinary albumin/urinary creatinine ratio (Alb/Cr). Lowdensity lipoprotein cholesterol (LDL-C) levels were calculated using the Friedewald formula. Creatinine clearance rate (Ccr) was calculated using the CockcroftGault formula.

Measurement of plasma BNP, corin and furin levels Venous blood samples were collected into ethylene- 
diaminetetraacetic acid (EDTA) - vacutainer tubes (BD diagnostics, Franklin Lakes, NJ, USA) by venipuncture. Plasma samples were immediately obtained, aliquoted in sterile polypropylene tubes and stored at $-80^{\circ} \mathrm{C}$ until analysis. Plasma levels of BNP, corin and furin were measured by a commercial enzyme-linked immunosorbent assay (ELISA; Abnova, Taipei, Taiwan) according to the manufacturer's instructions.

\section{Corin SNPs genotyping}

Two SNPs in exon 12 of corin gene (National center for biotechnology information reference sequence gene; NCBI RefSeq gene: NM_006587.2) at nucleotides 1757 C> T (rs75770792) and $1796 \mathrm{~A}>\mathrm{C}(\mathrm{rs} 111253292)$ were genotyped using allele specific oligonucleotidepolymerase chain reaction (ASO-PCR). Allele-specific primers were designed using "web-based allele-specific primer designing tool": http://bioinfo.biotec.or.th/WASP. The sequences of forward and reverse primers used for ASO-PCR are shown in Table 1. Briefly, genomic DNA was extracted from peripheral blood leukocytes using the Wizard ${ }^{\circledR}$ Genomic DNA Purification Kit (Promega, Madison, USA) following the manufacturer's instructions. PCR reactions were carried out in a total volume of $25 \mu \mathrm{L}$, containing $15 \mathrm{mM}$ Tris- $\mathrm{HCl}+50 \mathrm{mM} \mathrm{KCl}$ (Gene Amp 10X PCR Gold Buffer), $1.5 \mathrm{mM} \mathrm{MgCl}$ (Gene Amp 25 $\mathrm{mM} \mathrm{MgCl}$ ), $400 \mu \mathrm{M}$ of each dNTP (Gene Amp $10 \mathrm{mM}$ dNTP Blend), $1 \mathrm{U}$ Taq DNA polymerase (AmpliTaq Gold 5 U/ $\mu \mathrm{L}$ DNA polymerase) (Applied Biosystems, Foster City, CA, USA), $0.5 \mu \mathrm{M}$ of each of the primers (Sigma-Aldrich, St. Louis, MO, USA) and $100 \mathrm{ng}$ of genomic DNA. The thermal cycling conditions were as follows: initial denaturation at $94^{\circ} \mathrm{C}$ for 5 minutes followed by 35 amplification cycles of denaturation at $94^{\circ} \mathrm{C}$ for 30 seconds, annealing at $60^{\circ} \mathrm{C}$ for 30 seconds, and extension at $72^{\circ} \mathrm{C}$ for 30 seconds followed by terminal extension at $72^{\circ} \mathrm{C}$ for 10 minutes. The amplified products from ASO-PCR were electrophoresed on a 2\% agarose gel (Applied Biosystems/Ambion, Austin, USA) using GeneRuler
Ultra Low Range DNA Ladder (Thermo Scientific, Waltham, MA, USA) as a size marker, visualized by staining the gels with ethidium bromide, followed by destaining with water and finally photographed under Ultraviolet illumination using KODAK Gel Logic 100 Imaging System.

\section{Statistical analysis}

Categorical variables were reported as the number of cases (percentage) and compared using the Pearson's chisquare $\left(\chi^{2}\right)$ test. The continuous variables were expressed as mean \pm standard deviation (SD) if normally distributed and compared using the independent Student's t-test or one-way analysis of variance (ANOVA) as appropriate. In contrast, continuous variables were expressed as median (interquartile range, IQR: $25^{\text {th }}$ quartile to $75^{\text {th }}$ quartile or minimum-maximum as appropriate) if nonnormally distributed and compared using the nonparametric Mann-Whitney U test or Kruskal-Wallis test as appropriate. Normality was assessed by the ShapiroWilk test. A 2- sided probability $(P)$ value was used for all statistical analyses, and a $P$ value of $<0.05$ was considered statistically significant. The correlation of $\mathrm{BNP}$, corin and furin levels with continuous variables was analyzed using Spearman's rank correlation coefficient because data were not normally distributed. Receiver operating characteristic (ROC) curve analysis was performed to determine the diagnostic value of plasma BNP, corin and furin levels. Data statistical analyses were performed using the statistical package for the social sciences (SPSS software 20; SPSS Inc., Chicago, IL, USA) ${ }^{[25]}$. Genotypes and alleles frequency was estimated. Genotype frequencies at each corin gene locus were compared with the frequencies expected by the Hardy-Weinberg equilibrium (HWE) using a $\chi^{2}$ goodness of fit test. Pairwise linkage disequilibrium (LD) between the 2 corin gene loci was assessed by estimating the coefficients of $\mathrm{LD}\left(\mathrm{D}^{\prime}\right.$, Standardized disequilibrium; $\mathrm{r}^{2}$, Square of the correlation coefficient) ${ }^{[26]}$. All genetic analyses were performed using the genetic analysis in Excel 6.5 (GenAlEx 6.5) software ${ }^{[27,28]}$.

Table 1: The sequences of forward and reverse primers used for ASO-PCR.

\begin{tabular}{|c|c|c|c|c|c|}
\hline SNP & ref SNP ID & $\begin{array}{c}\text { Amino } \\
\text { acid } \\
\text { change }\end{array}$ & Primer type & $\begin{array}{c}\text { Primer sequence } \\
\left(\mathbf{5}^{\prime} \rightarrow \mathbf{3}^{\prime}\right)\end{array}$ & $\begin{array}{c}\text { PCR Product } \\
\text { length }(\mathbf{b p})\end{array}$ \\
\hline $\mathbf{1 7 5 7} \mathbf{C}>\mathbf{T}$ & rs75770792 & $\mathbf{T 5 5 5 I}$ & $\begin{array}{c}\text { WTF } \\
\text { MTF } \\
\text { CR }\end{array}$ & $\begin{array}{l}\text { CCTACAGTGGCCTGAAGACTC } \\
\text { CCTACAGTGGCCTGAAGACTT } \\
\text { AATTCACTCACCTAAGCAGC }\end{array}$ & 231 \\
\hline $\mathbf{1 7 9 6}$ A $>\mathbf{C}$ & $\mathbf{r s 1 1 1 2 5 3 2 9 2}$ & $\mathbf{Q 5 6 8 P}$ & $\begin{array}{c}\text { WTR } \\
\text { MTR }\end{array}$ & $\begin{array}{l}\text { CAGGCATCAGGCAGGTCT } \\
\text { CAGGCATCAGGCAGGTCG }\end{array}$ & 137 \\
& & & CF & ATCTAGGGCATTGTGTGAAC & \\
\hline
\end{tabular}

WTF, wild type forward; MTF, mutant type forward; CR, common reverse; WTR, wild type reverse; MTR, mutant type reverse; $\mathrm{CF}$, common forward. 


\section{Results}

Baseline characteristics of the study cohort

The study cohort was divided into 3 groups: 25 healthy subjects, 25 T2DM patients without CVDs and 25 T2DM patients with CVDs. Of the 25 patients with CVDs, 10 patients $(40 \%)$ had coronary artery disease (CAD), 7 patients $(28 \%)$ had myocardial infarction (MI), 5 patients $(20 \%)$ had chronic heart failure $(\mathrm{CHF})$ and 3 patients $(12 \%)$ had peripheral vascular disease (PVD). The baseline demographic and clinical features of the patients and healthy subjects are listed in Table 2.

Plasma levels of BNP, corin and furin in the study groups

BNP and furin levels were found to be significantly higher in T2DM patients with CVDs than those in T2DM patients without CVDs and those in healthy subjects. Furthermore, BNP and furin levels were higher in T2DM patients without CVDs than those in healthy subjects. On the other hand, corin levels appeared to be significantly higher in healthy subjects than those in T2DM patients with or without CVDs. Moreover, corin levels were higher in T2DM patients without CVDs than those in T2DM patients with CVDs (Table 2).

The plasma levels of BNP, corin and furin in the different CVD types are summarized in Table 3. There were no significant differences in BNP levels among patients with the different CVDs. One exception was that BNP levels were significantly higher in $\mathrm{CHF}$ patients than those in CAD patients. Similarly, there were no significant differences in corin levels among patients with the different CVDs. On the other hand, furin levels were significantly different among patients with the different CVDs. One exception was that furin levels in CHF patients did not differ significantly when compared to those in PVD patients.

Correlation of plasma BNP, corin and furin levels with other variables

BNP level was positively correlated with LDH and furin levels in healthy subjects. In T2DM patients with CVDs, BNP level was positively correlated with FPG, CK and CK-MB. Furthermore, there was a significant negative correlation between BNP and corin levels in T2DM patients with and without CVDs. In contrast, BNP level was positively correlated with furin level in T2DM patients with and without CVDs. On the other hand, a negative correlation was found between corin and furin levels in T2DM patients with CVDs, but this correlation did not meet the criteria for statistical significance (Table 4).

\section{ROC curve analysis}

The sensitivity of CVDs diagnosis in T2DM patients by furin level was significantly higher than that by corin. On the other hand, both furin and BNP have a similar sensitivity in diagnosing CVDs. Furthermore, in T2DM patients, the specificity of CVDs diagnosis by furin level was significantly higher than that by BNP or by corin. Differences in the positive predictive value (PPV), negative predictive value (NPV), accuracy, and area under the curve (AUC) between furin and corin were significant. In contrast, there were no significant differences in the PPV, NPV, accuracy, and AUC between furin and BNP (Table 5, Figure1).

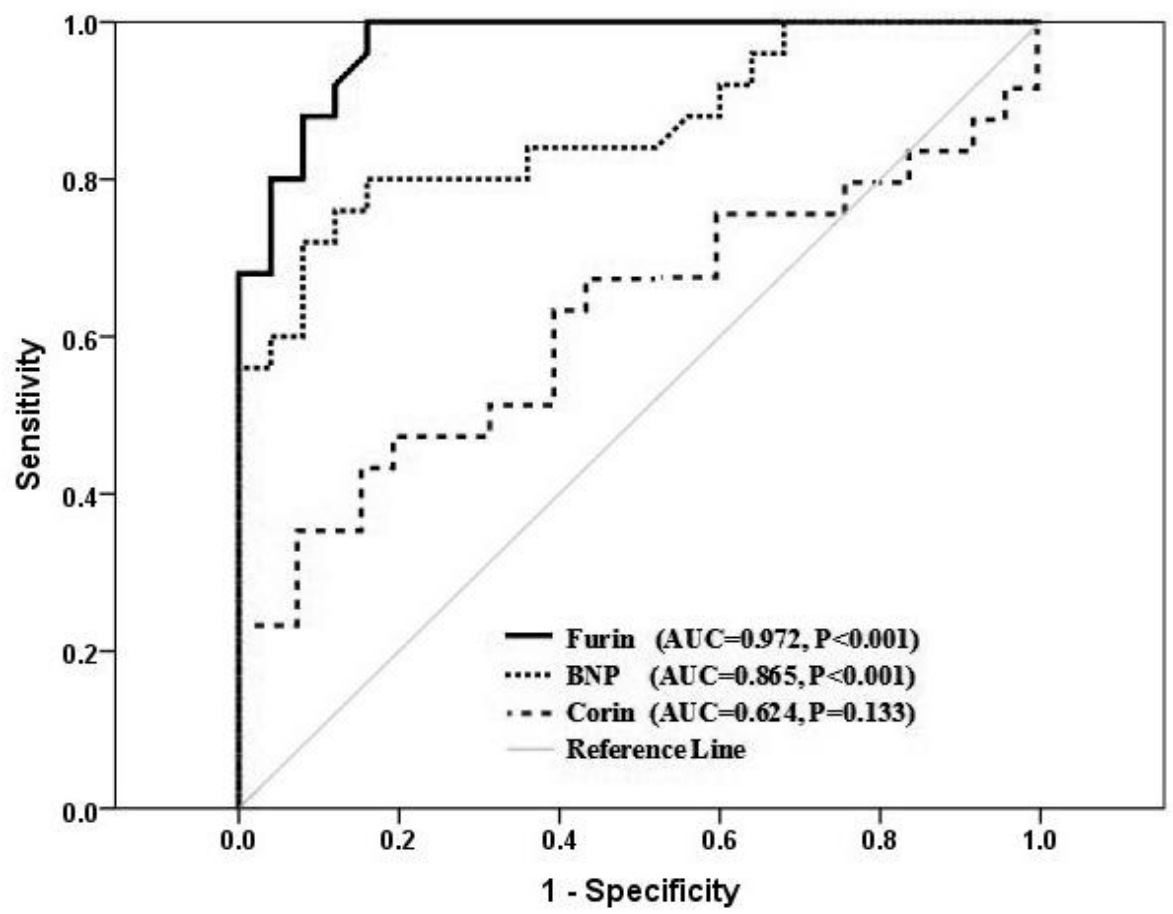

Fig. 1: Receiver operating characteristic (ROC) curve for predicting CVDs in T2DM patients. 
Table 2: Demographic and clinical characteristics of the study groups.

\begin{tabular}{|c|c|c|c|c|}
\hline \multirow[b]{2}{*}{ Characteristics } & \multicolumn{3}{|c|}{ Participants $(n=75)$} & \multirow[t]{2}{*}{$P$ value } \\
\hline & $\begin{array}{l}\text { Healthy subjects } \\
\qquad(\mathbf{n}=25)\end{array}$ & $\begin{array}{c}\text { T2DM } \\
\text { without CVDs }(n=25)\end{array}$ & $\begin{array}{c}\text { T2DM } \\
\text { With CVDs } \\
(\mathbf{n}=25)\end{array}$ & \\
\hline $\begin{array}{l}\text { Gender } \\
\text { Male, n (\%) } \\
\text { Female, n (\%) }\end{array}$ & $\begin{array}{l}13(52) \\
12(48)\end{array}$ & $\begin{array}{l}13(52) \\
12(48)\end{array}$ & $\begin{array}{l}13(52) \\
12(48)\end{array}$ & \\
\hline Age (years) & $\begin{array}{c}55.4 \pm 6.9 \\
(45-65)\end{array}$ & $\begin{array}{l}55.4 \pm 5.7 \\
(45-65)\end{array}$ & $\begin{array}{c}55.3 \pm 6.2 \\
(45-65)\end{array}$ & $\begin{array}{l}1.000^{\dagger} \\
1.000^{\ddagger} \\
1.000^{\S}\end{array}$ \\
\hline Age at onset of DM (years) & & $\begin{array}{c}46.5 \pm 6.7 \\
(31-60)\end{array}$ & $\begin{array}{c}42.8 \pm 9.5 \\
(26-55)\end{array}$ & $0.117^{\S}$ \\
\hline Age at onset of CVDs (years) & & & $\begin{array}{c}48.7 \pm 7.4 \\
(34-60)\end{array}$ & \\
\hline BMI $\left(\mathrm{kg} / \mathrm{m}^{2}\right)$ & $\begin{array}{c}29.3 \pm 6.0 \\
(20.1-43.1)\end{array}$ & $\begin{array}{l}29.5 \pm 4.4 \\
(23.2-40.4)\end{array}$ & $\begin{array}{c}30.0 \pm 3.9 \\
(23.2-39.4)\end{array}$ & $\begin{array}{l}1.000^{\dagger} \\
1.000^{\ddagger} \\
1.000^{\S}\end{array}$ \\
\hline $\begin{array}{l}\text { Cigarette smoking status } \\
\text { Non-smokers, } \mathrm{n}(\%) \\
\text { Past smokers, } \mathrm{n}(\%) \\
\text { Current smokers, } \mathrm{n}(\%) \\
\end{array}$ & $\begin{array}{l}17(68) \\
5(20) \\
3(12) \\
\end{array}$ & $\begin{array}{l}15(60) \\
5(20) \\
5(20) \\
\end{array}$ & $\begin{array}{l}13(52) \\
6(24) \\
6(24) \\
\end{array}$ & $\begin{array}{l}0.732^{\dagger} \\
0.444^{\ddagger} \\
0.850^{\S} \\
\end{array}$ \\
\hline $\begin{array}{l}\text { Family history of DM } \\
\text { Yes, } \mathrm{n}(\%) \\
\text { No, } \mathrm{n}(\%)\end{array}$ & $\begin{array}{c}0(0) \\
25(100)\end{array}$ & $\begin{array}{c}9(36) \\
16(64)\end{array}$ & $\begin{array}{l}16(64) \\
9(36)\end{array}$ & $\begin{array}{c}0.001^{\dagger} \\
<0.001^{\dagger *} \\
0.044^{\S *}\end{array}$ \\
\hline $\begin{array}{l}\text { Family history of CVDs } \\
\text { Yes, } \mathrm{n}(\%) \\
\text { No, } \mathrm{n}(\%)\end{array}$ & $\begin{array}{c}2(8) \\
23(92)\end{array}$ & $\begin{array}{c}1(4) \\
24(96) \\
\end{array}$ & $\begin{array}{r}8(32) \\
17(68) \\
\end{array}$ & $\begin{array}{c}0.500^{\dagger} \\
0.037^{\dagger *} \\
0.012^{\S *}\end{array}$ \\
\hline Diastolic blood pressure (mmHg) & $\begin{array}{c}79.2 \pm 4.5 \\
(70-85)\end{array}$ & $\begin{array}{c}84.0 \pm 10.1 \\
(65-100)\end{array}$ & $\begin{array}{c}92.4 \pm 12.4 \\
(75-130)\end{array}$ & $\begin{array}{c}0.244^{\dagger} \\
<0.001^{* *} \\
0.008^{\S *}\end{array}$ \\
\hline Systolic blood pressure (mmHg) & $\begin{array}{l}121.2 \pm 8.6 \\
(105-140)\end{array}$ & $\begin{array}{l}132.6 \pm 24.8 \\
(95-190)\end{array}$ & $\begin{array}{c}144.4 \pm 23.5 \\
(100-210)\end{array}$ & $\begin{array}{c}0.154^{\dagger} \\
<0.001^{+*} \\
0.132^{\S}\end{array}$ \\
\hline $\begin{array}{l}\text { Hypertension } \\
\text { Yes, n (\%) } \\
\text { No, n }(\%)\end{array}$ & $\begin{array}{c}0(0) \\
25(100)\end{array}$ & $\begin{array}{l}12(48) \\
13(52)\end{array}$ & $\begin{array}{l}15(60) \\
10(40)\end{array}$ & $\begin{array}{c}<0.001^{\dagger *} \\
<0.001^{\dagger *} \\
0.285^{\S}\end{array}$ \\
\hline $\begin{array}{l}\text { Asthma } \\
\text { Yes, n (\%) } \\
\text { No, n (\%) }\end{array}$ & $\begin{array}{c}0(0) \\
25(100)\end{array}$ & $\begin{array}{c}2(8) \\
23(92)\end{array}$ & $\begin{array}{c}6(24) \\
19(76)\end{array}$ & $\begin{array}{c}0.245^{\dagger} \\
0.011^{+*} \\
0.123^{\S}\end{array}$ \\
\hline $\begin{array}{l}\text { Nephropathy } \\
\text { Yes, n (\%) } \\
\text { No, n }(\%)\end{array}$ & $\begin{array}{c}0(0) \\
25(100)\end{array}$ & $\begin{array}{c}7(28) \\
18(72)\end{array}$ & $\begin{array}{l}11(44) \\
14(56)\end{array}$ & $\begin{array}{c}0.005^{\dagger *} \\
<0.001^{\dagger *} \\
0.189^{\S}\end{array}$ \\
\hline $\begin{array}{l}\text { Retinopathy } \\
\text { Yes, n }(\%) \\
\text { No, n }(\%)\end{array}$ & $\begin{array}{c}0(0) \\
25(100)\end{array}$ & $\begin{array}{c}3(12) \\
22(88)\end{array}$ & $\begin{array}{l}11(44) \\
14(56)\end{array}$ & $\begin{array}{c}0.117^{\dagger} \\
<0.001^{\dagger *} \\
0.013^{\S} *\end{array}$ \\
\hline $\begin{array}{l}\text { Neuropathy } \\
\text { Yes, n }(\%) \\
\text { No, n }(\%)\end{array}$ & $\begin{array}{c}0(0) \\
25(100)\end{array}$ & $\begin{array}{c}5(20) \\
20(80)\end{array}$ & $\begin{array}{l}13(52) \\
12(48)\end{array}$ & $\begin{array}{c}0.025^{\dagger *} \\
<0.001^{\dagger *} \\
0.019^{\S *}\end{array}$ \\
\hline $\begin{array}{l}\text { Recurrent infections } \\
\text { Yes, } \mathrm{n}(\%) \\
\text { No, n }(\%)\end{array}$ & $\begin{array}{c}0(0) \\
25(100)\end{array}$ & $\begin{array}{c}5(20) \\
20(80)\end{array}$ & $\begin{array}{c}2(8) \\
23(92)\end{array}$ & $\begin{array}{c}0.025^{\dagger *} \\
0.245^{\ddagger} \\
0.209^{\S}\end{array}$ \\
\hline Duration of DM (years) & & $\begin{array}{c}8.9 \pm 5.9 \\
(1-25)\end{array}$ & $\begin{array}{c}12.9 \pm 7.1 \\
(2-35)\end{array}$ & $0.035^{\$ *}$ \\
\hline $\begin{array}{l}\text { DM medications } \\
\text { Oral hypoglycemic agents, n (\%) } \\
\text { Insulin, n (\%) }\end{array}$ & & $\begin{array}{c}16(64) \\
9(36)\end{array}$ & $\begin{array}{c}9(36) \\
16(64)\end{array}$ & $0.044^{\S *}$ \\
\hline
\end{tabular}


Table 2 continued...

\begin{tabular}{|c|c|c|c|c|}
\hline $\begin{array}{l}\text { CVDs } \\
\text { CAD, n (\%) } \\
\text { MI, n (\%) } \\
\text { CHF, n (\%) } \\
\text { PVD, n (\%) }\end{array}$ & & & $\begin{array}{l}10(40) \\
7(28) \\
5(20) \\
3(12) \\
\end{array}$ & \\
\hline $\begin{array}{l}\text { CVDs medications } \\
\beta \text {-blockers + ACEI, n (\%) } \\
\beta \text {-blockers + ARB, n (\%) } \\
\end{array}$ & & & $\begin{array}{c}16(64) \\
9(36) \\
\end{array}$ & \\
\hline FPG (mg/dl) & $\begin{array}{c}84.5 \pm 9.0 \\
(69-108)\end{array}$ & $\begin{array}{c}166.3 \pm 55.6 \\
(105-360)\end{array}$ & $\begin{array}{c}208.2 \pm 74.3 \\
(110-459)\end{array}$ & $\begin{array}{l}<0.001^{\dagger *} \\
<0.001^{* *} \\
0.022^{\S *}\end{array}$ \\
\hline HbA1c \% $\pm(\mathrm{mmol} / \mathrm{mol})$ & $\begin{array}{c}5.0 \pm 0.5(31) \\
{[3.3-5.9]}\end{array}$ & $\begin{array}{c}6.2 \pm 1.3(44) \\
{[3.9-9.7]}\end{array}$ & $\begin{array}{c}7.4 \pm 1.5(57) \\
{[4.5-10.4]}\end{array}$ & $\begin{array}{c}0.002^{\uparrow *} \\
<0.001^{* *} \\
0.002^{\S *}\end{array}$ \\
\hline TC (mg/dl) & $\begin{array}{c}160.1 \pm 24.1 \\
(106-197)\end{array}$ & $\begin{array}{c}190.3 \pm 47.7 \\
(96-311)\end{array}$ & $\begin{array}{l}207 \pm 53.7 \\
(121-350)\end{array}$ & $\begin{array}{c}0.050^{\dagger} \\
0.001^{+*} \\
0.522 \S\end{array}$ \\
\hline HDL-C (mg/dl) & $\begin{array}{c}45.7 \pm 9.2 \\
(29-61)\end{array}$ & $\begin{array}{c}37.6 \pm 7.7 \\
(25-49)\end{array}$ & $\begin{array}{c}32.5 \pm 7.4 \\
(18-42)\end{array}$ & $\begin{array}{c}0.002^{\dagger *} \\
<0.001^{* *} \\
0.088^{\S}\end{array}$ \\
\hline LDL-C (mg/dl) & $\begin{array}{c}85.4 \pm 35.6 \\
(19-178)\end{array}$ & $\begin{array}{c}117.4 \pm 47.9 \\
(19-237)\end{array}$ & $\begin{array}{c}127.5 \pm 52.0 \\
(54-269)\end{array}$ & $\begin{array}{c}0.047^{\dagger *} \\
0.005^{\star *} \\
1.000^{\S}\end{array}$ \\
\hline TG (mg/dl) & $\begin{array}{c}144.7 \pm 67.3 \\
(85-393)\end{array}$ & $\begin{array}{c}176.3 \pm 69.7 \\
(77-381)\end{array}$ & $\begin{array}{c}233.7 \pm 90.9 \\
(107-464)\end{array}$ & $\begin{array}{c}0.449^{\dagger} \\
<0.001^{* *} \\
0.030^{\S *}\end{array}$ \\
\hline LDH (IU/L) & $\begin{array}{c}300.0 \\
(275.0-332.5) \\
\end{array}$ & $\begin{array}{c}335.0 \\
(290.5-400.5) \\
\end{array}$ & $\begin{array}{c}414.0 \\
(322.0-674.0) \\
\end{array}$ & $\begin{array}{l}0.052^{\dagger} \\
0.001^{*^{*}} \\
0.026^{\S^{*}}\end{array}$ \\
\hline CK (IU/L) & $\begin{array}{c}80.0 \\
(73.5-96.0)\end{array}$ & $\begin{array}{c}120.0 \\
(77.0-168.0)\end{array}$ & $\begin{array}{c}178.0 \\
(122.5-395.0)\end{array}$ & $\begin{array}{c}0.023^{T^{+*}} \\
<0.001^{\text {t* }^{*}} \\
0.002^{\S_{*}}\end{array}$ \\
\hline CK-MB (IU/L) & $\begin{array}{c}9.0 \\
(7.5-10.0)\end{array}$ & $\begin{array}{c}12.0 \\
(9.5-15.5)\end{array}$ & $\begin{array}{c}17.0 \\
(15.0-42.5)\end{array}$ & $\begin{array}{c}0.002^{\uparrow^{*}} \\
<0.001^{\text {t* }^{*}} \\
<0.001^{\text {\&* }}\end{array}$ \\
\hline UA (mg/dl) & $\begin{array}{c}4.3 \pm 0.80 \\
(3.2-6.0)\end{array}$ & $\begin{array}{l}5.0 \pm 1.1 \\
(3.5-8.7)\end{array}$ & $\begin{array}{c}5.7 \pm 2.5 \\
(2.4-11.9)\end{array}$ & $\begin{array}{c}0.592^{\dagger} \\
0.017^{* *} \\
0.372^{\S}\end{array}$ \\
\hline $\mathrm{Cr}(\mathrm{mg} / \mathrm{dl})$ & $\begin{array}{l}0.75 \pm 0.22 \\
(0.30-1.03)\end{array}$ & $\begin{array}{l}0.99 \pm 0.33 \\
(0.29-1.80)\end{array}$ & $\begin{array}{l}1.25 \pm 0.43 \\
(0.7-2.10)\end{array}$ & $\begin{array}{c}0.034^{\dagger *} \\
<0.001^{* *} \\
0.024^{\S *}\end{array}$ \\
\hline Urinary Alb (mg/L) & $\begin{array}{c}19.5 \\
(10.5-38.5)\end{array}$ & $\begin{array}{c}70.0 \\
(22.5-307.0)\end{array}$ & $\begin{array}{c}199.0 \\
(20.5-779.5)\end{array}$ & $\begin{array}{c}0.001^{\dagger *} \\
<0.001^{\ddagger *} \\
0.146^{\S}\end{array}$ \\
\hline Urinary $\mathrm{Cr}$ (mg/dl) & $\begin{array}{c}199.2 \\
(146.3-292.8)\end{array}$ & $\begin{array}{c}103.2 \\
(67.8-120.1)\end{array}$ & $\begin{array}{c}103.0 \\
(57.8-131.2)\end{array}$ & $\begin{array}{c}<0.001^{\dagger *} \\
<0.001^{\ddagger} \\
0.786^{\S}\end{array}$ \\
\hline Urinary $\mathrm{Alb} / \mathrm{Cr}$ ratio $(\mathrm{mg} / \mathrm{g})$ & $\begin{array}{c}11.6 \\
(5.2-18.0)\end{array}$ & $\begin{array}{c}44.2 \\
(23.9-449.0)\end{array}$ & $\begin{array}{c}152.7 \\
(50.7-696.5)\end{array}$ & $\begin{array}{c}<0.001^{\uparrow} \\
<0.001^{+*} \\
0.028^{\S *}\end{array}$ \\
\hline $\operatorname{Ccr}(\mathrm{ml} / \mathrm{min})$ & $\begin{array}{c}118.6 \\
(99.1-163.7)\end{array}$ & $\begin{array}{c}91.0 \\
(71.3-120.9)\end{array}$ & $\begin{array}{c}77.7 \\
(56.5-96.9)\end{array}$ & $\begin{array}{c}0.013^{\uparrow *} \\
<0.001^{* *} \\
0.056^{\S}\end{array}$ \\
\hline BNP (pg/ml) & $\begin{array}{c}30.7 \\
(18.4-45.2)\end{array}$ & $\begin{array}{c}69.2 \\
(44.1-92.5)\end{array}$ & $\begin{array}{c}430.3 \\
(127.8-818.5)\end{array}$ & $\begin{array}{l}<0.001^{\dagger *} \\
<0.001^{* *} \\
<0.001^{\S *}\end{array}$ \\
\hline Corin (pg/ml) & $\begin{array}{c}1325.0 \\
(693.5-1724.5)\end{array}$ & $\begin{array}{c}612.0 \\
(375.0-1096.5)\end{array}$ & $\begin{array}{c}489.0 \\
(337.5-633.5)\end{array}$ & $\begin{array}{c}0.004^{\dagger *} \\
<0.001^{* *} \\
0.133^{\S}\end{array}$ \\
\hline Furin (pg/ml) & $\begin{array}{c}157.3 \\
(130.9-184.5)\end{array}$ & $\begin{array}{c}185.5 \\
(145.5-221.9)\end{array}$ & $\begin{array}{c}1046.9 \\
(458.1-1716.2)\end{array}$ & $\begin{array}{c}0.074^{\dagger} \\
<0.001^{+*} \\
<0.001^{\S *}\end{array}$ \\
\hline
\end{tabular}

T2DM, type 2 diabetes mellitus; BMI, body mass index; CVDs, cardiovascular diseases; CAD: coronary artery disease; MI, myocardial infarction; CHF, chronic heart failure; PVD: peripheral vascular disease; ACEI, angiotensin-converting enzyme inhibitor; ARB, angiotensinreceptor blocker; FPG, fasting plasma glucose; HbA1c, hemoglobin A1c; TC, total cholesterol; HDL-C, high-density lipoprotein cholesterol; LDL-C, low-density lipoprotein cholesterol; TG, triglycerides; LDH, lactate dehydrogenase; CK, creatine kinase; UA, uric acid; Cr, creatinine; Alb, albumin; Ccr, creatinine clearance rate; BNP, brain natriuretic peptide. Qualitative data are represented as the number of cases (\%), whereas quantitative data are represented as mean $\pm \mathrm{SD}$ (range, minimum-maximum) if normally distributed or as median (interquartile range, IQR: $25^{\text {th }}$ quartile to $75^{\text {th }}$ quartile) if non-normally distributed. $\dagger$, healthy subjects vs. T2DM without CVDs; $\$$, healthy subjects vs. T2DM with CVDs; $\S$, T2DM without CVDs vs. T2DM with CVDs. * indicates a statistically significant difference. 
Table 3: Relationship of BNP, corin and furin levels with the different CVD types.

\begin{tabular}{|c|c|c|c|c|c|}
\hline \multirow{2}{*}{$\underset{(\mathbf{p g} / \mathrm{ml})}{\text { Biomarker level }}$} & \multicolumn{4}{|c|}{$\begin{array}{l}\text { T2DM with CVDs (n=25) } \\
\text { n (\%) }\end{array}$} & \multirow[t]{2}{*}{$P$ value } \\
\hline & $\begin{array}{c}\text { CAD } \\
10(40)\end{array}$ & $\underset{7(28)}{\text { MI }}$ & $\begin{array}{l}\text { CHF } \\
5(20)\end{array}$ & $\begin{array}{l}\text { PVD } \\
3(12)\end{array}$ & \\
\hline BNP & $\begin{array}{c}98.3 \\
(59.1-447.4)\end{array}$ & $\begin{array}{c}192.8 \\
(167.4-817.5)\end{array}$ & $\begin{array}{c}823.0 \\
(601.2-1219.8)\end{array}$ & $\begin{array}{c}679.5 \\
(633.3-820.2)\end{array}$ & $\begin{array}{c}0.055^{\text {II }} \\
0.008^{Q_{*}^{*}} \\
0.112^{\Upsilon} \\
0.073^{\mathrm{W}} \\
0.267^{*} \\
0.393^{\mathrm{x}}\end{array}$ \\
\hline Corin & $\begin{array}{c}604.2 \\
(450.5-824.3)\end{array}$ & $\begin{array}{c}512.0 \\
(361.0-653.0)\end{array}$ & $\begin{array}{c}314.0 \\
(210.5-558.0)\end{array}$ & $\begin{array}{c}409.0 \\
(233-489.0)\end{array}$ & $\begin{array}{l}0.669^{I I} \\
0.129^{\mathrm{Q}} \\
0.217^{\Upsilon} \\
0.202^{\mathrm{WI}} \\
0.383^{\text {}} \\
1.000^{x}\end{array}$ \\
\hline Furin & $\begin{array}{c}385.0 \\
(265.4-562.5)\end{array}$ & $\begin{array}{c}1046.9 \\
(814.5-1436.2)\end{array}$ & $\begin{array}{c}2156.7 \\
(1664.6-2503)\end{array}$ & $\begin{array}{c}1719.1 \\
(1713.2-1769.5)\end{array}$ & 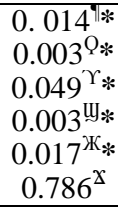 \\
\hline
\end{tabular}

T2DM, type 2 diabetes mellitus; CVDs, cardiovascular diseases; CAD: coronary artery disease; MI, myocardial infarction; CHF, chronic heart failure; PVD: peripheral vascular disease; BNP, brain natriuretic peptide. Qualitative data are represented as the number of cases $(\%)$, whereas quantitative data are represented as median (interquartile range, IQR: $25^{\text {th }}$ quartile to $75^{\text {th }}$ quartile or minimum-maximum as appropriate). II, CAD vs. MI; Q, CAD vs. CHF; ' $\Upsilon$, CAD vs. PVD; W, MI vs. CHF; Ж, MI vs. PVD; $\Upsilon$, CHF vs. PVD. * indicates a statistically significant difference.

Table 4: Correlation of plasma BNP, corin and furin levels with other variables in the study groups.

\begin{tabular}{|c|c|c|c|c|c|c|c|c|c|c|c|c|c|c|c|c|c|c|}
\hline \multirow[t]{3}{*}{ Variables } & \multicolumn{6}{|c|}{ Plasma BNP level (pg/ml) } & \multicolumn{6}{|c|}{ Plasma corin level (pg/ml) } & \multicolumn{6}{|c|}{ Plasma furin level (pg/ml) } \\
\hline & \multicolumn{2}{|c|}{$\begin{array}{c}\text { Healthy } \\
\text { subjects } \\
(n=25)\end{array}$} & \multicolumn{2}{|c|}{\begin{tabular}{|c|} 
T2DM \\
without CVDs \\
$(n=25)$
\end{tabular}} & \multicolumn{2}{|c|}{$\begin{array}{c}\text { T2DM } \\
\text { With CVDs } \\
(\mathbf{n}=25)\end{array}$} & \multicolumn{2}{|c|}{$\begin{array}{l}\text { Healthy } \\
\text { subjects } \\
(n=25)\end{array}$} & \multicolumn{2}{|c|}{$\begin{array}{c}\text { T2DM } \\
\text { without CVDs } \\
(n=25)\end{array}$} & \multicolumn{2}{|c|}{$\begin{array}{c}\text { T2DM } \\
\text { With CVDs } \\
(\mathbf{n}=25)\end{array}$} & \multicolumn{2}{|c|}{$\begin{array}{c}\text { Healthy } \\
\text { subjects } \\
(n=25)\end{array}$} & \multicolumn{2}{|c|}{$\begin{array}{c}\text { T2DM } \\
\text { without CVDs } \\
(\mathbf{n}=25)\end{array}$} & \multicolumn{2}{|c|}{$\begin{array}{c}\text { T2DM } \\
\text { With CVDs } \\
(n=25)\end{array}$} \\
\hline & $\mathbf{R}$ & $P$ value & $\mathbf{R}$ & $P$ value & $\mathbf{R}$ & $P$ value & $\mathbf{R}$ & $P$ value & $\mathbf{R}$ & $P$ value & $\mathbf{R}$ & $P$ value & $\mathbf{R}$ & $P$ value & $\mathbf{R}$ & $P$ value & $\mathbf{R}$ & $P$ value \\
\hline Age & 0.082 & 0.696 & -0.009 & 0.966 & 0.057 & 0.788 & 0.078 & 0.710 & -0.288 & 0.162 & -0.296 & 0.151 & -0.080 & 0.704 & -0.145 & 0.489 & -0.042 & 0.840 \\
\hline BMI & 0.138 & 0.511 & 0.160 & 0.446 & 0.130 & 0.535 & 0.037 & 0.862 & -0.153 & 0.465 & -0.201 & 0.336 & 0.207 & 0.321 & 0.035 & 0.867 & 0.332 & 0.105 \\
\hline FPG & 200 & 0.338 & 0.005 & 0.981 & 0.437 & $0.029^{*}$ & .248 & 0.232 & -0.085 & 0.685 & -0.117 & 0.579 & -0.053 & 0.803 & 0.173 & 0.409 & 0.095 & 0.651 \\
\hline HbA1c & 0.020 & 0.926 & 0.201 & 0.335 & 0.296 & 0.151 & 0.081 & 0.699 & -0.313 & 0.128 & 0.015 & 0.943 & 0.094 & 0.655 & 0.228 & 0.273 & 0.128 & 0.542 \\
\hline TC & -0.148 & 0.479 & 0.094 & 0.654 & 0.119 & 0.570 & -0.207 & 0.321 & 0.220 & 0.291 & -0.3 & 0.145 & -0.398 & 0.051 & 0.033 & 0.874 & 0.021 & 0.922 \\
\hline HDL-C & 0.225 & 0.280 & 0.300 & 0.145 & -0.056 & 0.789 & 0.067 & 0.749 & -0.379 & 0.061 & 0.139 & 0.508 & 0.331 & 0.106 & 0.110 & 0.600 & 0.222 & 0.286 \\
\hline LDL-C & -0.125 & 0.551 & -0.043 & 0.839 & 0.123 & 0.558 & -0.346 & 0.090 & 0.285 & 0.167 & -0.227 & 0.186 & -0.062 & 0.768 & -0.155 & 0.460 & -0.015 & 0.945 \\
\hline TG & -0.374 & 0.066 & 0.280 & 0.176 & -0.098 & 0.641 & -0.065 & 0.756 & 0.001 & 0.997 & -0.208 & 0.318 & -0.235 & 0.258 & 0.385 & 0.058 & 0.145 & 0.491 \\
\hline LDH & 0.494 & $0.012 *$ & -0.246 & 0.235 & 0.373 & 0.066 & -0.057 & 0.788 & 0.133 & 0.526 & -0.257 & 0.216 & 0.219 & 0.294 & -0.075 & 0.723 & 0.081 & 0.702 \\
\hline CK & 0.126 & 0.549 & -0.090 & 0.668 & 0.531 & $0.006^{*}$ & 0.211 & 0.310 & 0.155 & 0.460 & -0.110 & 0.599 & 0.026 & 0.902 & -0.080 & 0.705 & 0.242 & 0.243 \\
\hline CK-MB & 0.106 & 0.613 & -0.080 & 0.705 & 0.551 & 0.004* & 0.225 & 0.279 & 0.086 & 0.684 & -0.238 & 0.251 & 0.067 & 0.750 & 0.039 & 0.855 & 0.139 & 0.508 \\
\hline UA & 0.138 & 0.511 & -0.146 & 0.487 & 0.066 & 0.755 & -0.038 & 0.857 & -0.011 & 0.958 & 0.121 & 0.563 & -0.041 & 0.846 & -0.271 & 0.189 & 0.078 & 0.712 \\
\hline $\mathrm{Cr}$ & 0.073 & 0.728 & 0.076 & 0.718 & -0.032 & 0.879 & -0.066 & 0.755 & -0.277 & 0.181 & -0.038 & 0.858 & 0.168 & 0.421 & -0.251 & 0.226 & 0.177 & 0.398 \\
\hline $\begin{array}{c}\text { Urinary } \\
\text { Alb } \\
\end{array}$ & 0.310 & 0.131 & 0.241 & 0.245 & 0.151 & 0.472 & -0.004 & 0.983 & 0.052 & 0.805 & -0.107 & 0.612 & 0.099 & 0.638 & 0.162 & 0.439 & 0.035 & 0.867 \\
\hline Urinary $\mathbf{C r}$ & -0.025 & 0.906 & -0.052 & 0.807 & -0.036 & 0.865 & -0.029 & 0.891 & -0.095 & 0.653 & 0.046 & 0.828 & 0.283 & 0.170 & 0.030 & 0.885 & 0.036 & 0.864 \\
\hline $\begin{array}{c}\text { Urinary } \\
\text { Alb/Cr ratio } \\
\end{array}$ & 0.336 & 0.101 & 0.219 & 0.294 & 0.171 & 0.414 & -0.128 & 0.543 & 0.114 & 0.588 & -0.214 & 0.304 & -0.078 & 0.711 & 0.090 & 0.667 & -0.033 & 0.874 \\
\hline Cer & 0.202 & 0.333 & 0.060 & 0.774 & -0.015 & 0.942 & 0.119 & 0.573 & 0.145 & 0.490 & 0.173 & 0.408 & 0.035 & 0.867 & 0.247 & 0.234 & -0.081 & 0.701 \\
\hline BNP & - & - & - & - & - & - & -0.021 & 0.922 & -0.558 & 0.004* & -0.453 & $0.023 *$ & 0.494 & $0.012^{*}$ & 0.587 & 0.002* & 0.709 & $<0.001^{*}$ \\
\hline Corin & -0.021 & 0.922 & -0.558 & 0.004* & -0.453 & $0.023^{*}$ & - & - & - & - & - & - & 0.152 & 0.468 & -0.319 & 0.120 & -0.344 & 0.092 \\
\hline Furin & 0.494 & $0.012 *$ & 0.587 & $0.002 *$ & 0.709 & $<0.001 *$ & 0.152 & 0.468 & -0.319 & 0.120 & -0.344 & 0.092 & - & - & - & - & - & - \\
\hline
\end{tabular}

T2DM, type 2 diabetes mellitus; BMI, body mass index; CVDs, cardiovascular diseases; FPG, fasting plasma glucose; HbA1c, hemoglobin A1c; TC, total cholesterol; HDL-C, high-density lipoprotein cholesterol; LDL-C, low-density lipoprotein cholesterol; TG, triglycerides; LDH, lactate dehydrogenase; $\mathrm{CK}$, creatine kinase; UA, uric acid; $\mathrm{Cr}$, creatinine; Alb, albumin; Ccr, creatinine clearance rate; BNP, brain natriuretic peptide. The correlation was analyzed using Spearman's rank correlation coefficient. * indicates a statistically significant correlation. 
Table 5: Youden index cut-off points, sensitivity, specificity, positive predictive value, negative predictive value and area under the curve of BNP, corin and furin for predicting CVDs in T2DM patients.

\begin{tabular}{|c|c|c|c|c|c|c|c|c|c|c|}
\hline Biomarker & $\begin{array}{c}\text { T2DM } \\
\text { without } \\
\text { CVDs } \\
(\mathbf{n}=25)\end{array}$ & $\begin{array}{c}\text { T2DM } \\
\text { with } \\
\text { CVDs } \\
(\mathbf{n}=25)\end{array}$ & $\begin{array}{l}\text { Cut-off } \\
\text { point } \\
(\mathbf{p g} / \mathrm{ml})\end{array}$ & $\begin{array}{c}\text { Sensitivity } \\
(\%)\end{array}$ & $\begin{array}{c}\text { Specificity } \\
(\%)\end{array}$ & $\begin{array}{l}\text { PPV } \\
(\%)\end{array}$ & $\begin{array}{l}\text { NPV } \\
(\%)\end{array}$ & $\begin{array}{c}\text { Accuracy } \\
(\%)\end{array}$ & $\begin{array}{c}\text { AUC } \\
(95 \% \mathrm{CI})\end{array}$ & $P$ value \\
\hline \multirow{2}{*}{ BNP } & 21 & 5 & $\leq 109.4$ & \multirow{2}{*}{80.0} & \multirow{2}{*}{84.0} & \multirow{2}{*}{83.3} & \multirow{2}{*}{80.8} & \multirow{2}{*}{82.0} & \multirow{2}{*}{$\begin{array}{c}0.865 \\
(0.763-0.967)\end{array}$} & \multirow{2}{*}{$<0.001 *$} \\
\hline & 4 & 20 & $>109.4$ & & & & & & & \\
\hline \multirow{2}{*}{ Corin } & 9 & 14 & $\leq 501$ & \multirow{2}{*}{56.0} & \multirow{2}{*}{64.0} & \multirow{2}{*}{60.9} & \multirow{2}{*}{59.3} & \multirow{2}{*}{60.0} & \multirow{2}{*}{$\begin{array}{c}0.624 \\
(0.464-0.784)\end{array}$} & \multirow{2}{*}{0.133} \\
\hline & 16 & 11 & $>501$ & & & & & & & \\
\hline \multirow{2}{*}{ Furin } & 24 & 5 & $\leq 405.4$ & \multirow{2}{*}{80} & \multirow{2}{*}{96.0} & \multirow{2}{*}{92.5} & \multirow{2}{*}{82.5} & \multirow{2}{*}{88.0} & \multirow{2}{*}{$\begin{array}{c}0.972 \\
(0.935-1.00)\end{array}$} & \multirow{2}{*}{$<0.001 *$} \\
\hline & 1 & 20 & $>405.4$ & & & & & & & \\
\hline \multicolumn{4}{|c|}{$P$ value } & $\begin{array}{l}0.008^{\dagger *} \\
1.000^{\ddagger} \\
0.008^{\S *}\end{array}$ & $\begin{array}{l}0.019^{\dagger *} \\
0.041^{* *} \\
<0.001^{\S *}\end{array}$ & $\begin{array}{c}0.010^{\dagger *} \\
0.154^{\ddagger} \\
<0.001^{1^{\sharp} *}\end{array}$ & $\begin{array}{c}0.016^{\dagger *} \\
0.826^{\ddagger} \\
0.008^{\S *}\end{array}$ & $\begin{array}{c}0.012^{\dagger *} \\
0.399^{*} \\
0.001^{\S *}\end{array}$ & $\begin{array}{c}0.006^{\dagger *} \\
0.066^{\ddagger} \\
<0.001^{\S *}\end{array}$ & \\
\hline
\end{tabular}

T2DM, type 2 diabetes mellitus; CVDs, cardiovascular diseases; BNP, brain natriuretic peptide; PPV, Positive predictive value; NPV, Negative predictive value; AUC, area under the curve; $95 \%$ CI, $95 \%$ confidence interval. $\dagger$, BNP vs. corin; + , BNP vs. furin; $\S$, Corin vs. furin.

* indicates a statistically significant difference.

Table 6: Genotype distributions and allele frequencies of corin gene SNPs in the study groups.

\begin{tabular}{|c|c|c|c|c|c|c|}
\hline \multirow{2}{*}{\multicolumn{2}{|c|}{$\begin{array}{l}\text { Locus: C1757T } \\
\text { ref SNP ID: rs75770792 }\end{array}$}} & \multicolumn{3}{|c|}{$\begin{array}{c}\text { Genotype distribution } \\
n(\%)\end{array}$} & \multicolumn{2}{|c|}{$\begin{array}{l}\text { Allele frequency } \\
(\%)\end{array}$} \\
\hline & & $\mathrm{C} / \mathrm{C}$ & $\mathrm{C} / \mathrm{T}$ & $\mathbf{T} / \mathbf{T}$ & $\mathrm{C}$ allele & $T$ allele \\
\hline \multicolumn{2}{|c|}{ Subjects $(n=75)$} & $\begin{array}{c}74 \\
(98.67 \%)\end{array}$ & $\begin{array}{c}1 \\
(1.33 \%)\end{array}$ & $\begin{array}{c}0 \\
(0 \%)\end{array}$ & $99.3 \%$ & $0.7 \%$ \\
\hline \multirow{3}{*}{ Groups } & $\begin{array}{l}\text { Healthy subjects } \\
(n=25)\end{array}$ & $\begin{array}{c}25 \\
(100 \%)\end{array}$ & $\begin{array}{c}0 \\
(0 \%)\end{array}$ & $\begin{array}{c}0 \\
(0 \%)\end{array}$ & $100 \%$ & $0 \%$ \\
\hline & $\begin{array}{l}\text { T2DM without CVDs } \\
(\mathrm{n}=25)\end{array}$ & $\begin{array}{c}25 \\
(100 \%)\end{array}$ & $\begin{array}{c}0 \\
(0 \%)\end{array}$ & $\begin{array}{c}0 \\
(0 \%)\end{array}$ & $100 \%$ & $0 \%$ \\
\hline & $\begin{array}{l}\text { T2DM with CVDs } \\
(\mathbf{n}=25)\end{array}$ & $\begin{array}{c}24 \\
(96 \%)\end{array}$ & $\begin{array}{c}1 \\
(4 \%)\end{array}$ & $\begin{array}{c}0 \\
(0 \%)\end{array}$ & $98 \%$ & $2 \%$ \\
\hline \multicolumn{2}{|r|}{$P$ value } & $\begin{array}{l}1.000^{\dagger} \\
0.500^{\ddagger} \\
0.500^{\S}\end{array}$ & $\begin{array}{l}1.000^{\dagger} \\
0.500^{\ddagger} \\
0.500^{\S}\end{array}$ & & $\begin{array}{l}1.000^{\dagger} \\
0.500^{\ddagger} \\
0.500^{\S}\end{array}$ & $\begin{array}{l}1.000^{\dagger} \\
0.500^{\ddagger} \\
0.500^{\S}\end{array}$ \\
\hline \multirow{2}{*}{\multicolumn{2}{|c|}{$\begin{array}{l}\text { Locus: A1796C } \\
\text { ref SNP ID: rs111253292 }\end{array}$}} & \multicolumn{3}{|c|}{$\begin{array}{c}\text { Genotype distribution } \\
\text { n (\%) }\end{array}$} & \multicolumn{2}{|c|}{$\begin{array}{c}\text { Allele frequency } \\
(\%)\end{array}$} \\
\hline & & $\mathbf{A} / \mathbf{A}$ & $\mathrm{A} / \mathrm{C}$ & $\mathrm{C} / \mathrm{C}$ & A allele & C allele \\
\hline \multicolumn{2}{|c|}{ Subjects $(n=75)$} & $\begin{array}{c}74 \\
(98.67 \%)\end{array}$ & $\begin{array}{c}1 \\
(1.33 \%)\end{array}$ & $\begin{array}{c}0 \\
(0 \%)\end{array}$ & $99.3 \%$ & $0.7 \%$ \\
\hline \multirow{3}{*}{ Groups } & $\begin{array}{l}\text { Healthy subjects } \\
(n=25)\end{array}$ & $\begin{array}{c}25 \\
(100 \%)\end{array}$ & $\begin{array}{c}0 \\
(0 \%)\end{array}$ & $\begin{array}{c}0 \\
(0 \%)\end{array}$ & $100 \%$ & $0 \%$ \\
\hline & $\begin{array}{l}\text { T2DM without CVDs } \\
(\mathbf{n}=25)\end{array}$ & $\begin{array}{c}25 \\
(100 \%)\end{array}$ & $\begin{array}{c}0 \\
(0 \%)\end{array}$ & $\begin{array}{c}0 \\
(0 \%)\end{array}$ & $100 \%$ & $0 \%$ \\
\hline & $\begin{array}{l}\text { T2DM with CVDs } \\
(\mathbf{n}=25)\end{array}$ & $\begin{array}{c}24 \\
(96 \%)\end{array}$ & $\begin{array}{c}1 \\
(4 \%)\end{array}$ & $\begin{array}{c}0 \\
(0 \%)\end{array}$ & $98 \%$ & $2 \%$ \\
\hline \multicolumn{2}{|r|}{$P$ value } & $\begin{array}{l}1.000^{\dagger} \\
0.500^{\ddagger} \\
0.500^{\S}\end{array}$ & $\begin{array}{l}1.000^{\dagger} \\
0.500^{\ddagger} \\
0.500^{\S}\end{array}$ & & $\begin{array}{l}1.000^{\dagger} \\
0.500^{\ddagger} \\
0.500^{\S}\end{array}$ & $\begin{array}{l}1.000^{\dagger} \\
0.500^{\ddagger} \\
0.500^{\S}\end{array}$ \\
\hline
\end{tabular}

SNP, single nucleotide polymorphism; T2DM, type 2 diabetes mellitus; CVDs, cardiovascular diseases. $\dagger$, healthy subjects vs. T2DM without CVDs; $\$$, healthy subjects vs. T2DM with CVDs; §, T2DM without CVDs vs. T2DM with CVDs. 


\section{Corin gene SNPs}

Genotype distributions and allele frequencies of corin SNPs at 1757 and 1796 loci are presented in Table 6. The genotype distributions and allele frequencies of C1757T and A1796C SNPs did not differ significantly among the different study groups. The distribution of the observed genotypes was not significantly different from the expected distribution according to HWE for both loci $\left(\chi^{2}=0.003, P=0.954\right)$. The two SNPs (C1757T and A1796C) showed a significant $\left(\mathrm{D}^{\prime}=149 ; P<0.001\right)$ and strong $\left(r^{2}=1\right) L D$ to each other.

Relationship between plasma corin levels and corin gene SNPs

Corin gene SNPs at both loci (1757 and 1796) are not associated with plasma corin levels neither in the study cohort nor in T2DM patients with CVDs. In the study cohort, the median corin level was $644.5 \mathrm{pg} / \mathrm{ml}(\mathrm{IQR}=$ $411.8-1292.8 \mathrm{pg} / \mathrm{ml}$ ) in participants with $1757 \mathrm{CC}$ and $1796 \mathrm{AA}$, whereas the only participant with $1757 \mathrm{CT}$ and $1796 \mathrm{AC}$ had a corin level of $653 \mathrm{pg} / \mathrm{ml} \quad(P=0.960)$. Similarly, in T2DM patients with CVDs, the median corin level was $484.3 \mathrm{pg} / \mathrm{ml}(\mathrm{IQR}=325.7-623.5 \mathrm{pg} / \mathrm{ml})$ in patients with 1757CC and 1796AA, whereas the only patient with $1757 \mathrm{CT}$ and $1796 \mathrm{AC}$ had a corin level of $653 \mathrm{pg} / \mathrm{ml}(P=0.400)$.

\section{Discussion}

The incidence of diabetes is escalating worldwide and, consequently, this has become a major health care problem. Moreover, T2DM is associated with significantly accelerated rates of microvascular and macrovascular complications. However, the molecular mechanisms underlying the high incidence of diabetic complications are still not fully understood. When cardiovascular complications progress to an end stage, medical options are limited. Thus, timely diagnosis and early intervention are important for managing these life-threatening diseases.

BNP is a cardiac hormone, released from the cardiac ventricles in response to increased myocardial stretch or wall tension ${ }^{[29]}$. When ventricular myocytes secrete pro$\mathrm{BNP}_{108}$, furin, corin or other currently unknown proteases are thought to cleave pro- $\mathrm{BNP}_{108}$ to pro$\mathrm{BNP}_{77-108}$ (BNP-32), which is considered to be the biologically active hormone and an inactive amino terminal pro-BNP ${ }_{1-76}\left(\mathrm{NT}-\right.$ pro-BNP-76) ${ }^{[30]}$.

In the current study, BNP levels were found to be significantly higher in T2DM patients than in healthy subjects. Our results are consistent with previous findings that suggested a close relationship between glucose metabolism and BNP levels. These findings demonstrated that BNP levels were found to be higher in T2DM patients ${ }^{[31]}$. Our results showed that BNP levels were significantly higher in T2DM patients with CVDs when compared to those in T2DM patients without CVDs. Recently, Jin et al. have demonstrated that BNP levels were markedly higher in T2DM patients with peripheral arterial disease (PAD) than those in T2DM patients without PAD ${ }^{[32]}$. It has been previously demonstrated that plasma BNP levels predicted the risk of cardiovascular events and death after adjustment for clinical risk factors ${ }^{[33]}$. Moreover, it has been acknowledged that subjects with higher BNP levels are more likely to have conventional cardiovascular risk factors (e.g., hypertension ${ }^{[34]}$, and ischemic stroke ${ }^{[35]}$ ). In patients with end-stage HF, plasma pro-BNP levels were found to be elevated, suggesting that processing of this peptide is compromised as the disease progresses. The increased pro-BNP levels in severe HF may be explained in part by the increased production and secretion of pro-BNP from the ventricle. Alternatively, the mRNA expression of proteolytic processing enzymes is not increased in parallel with the increase in mRNA expression of BNP precursors in severe HF, which results in the reduced proteolytic conversion of pro-BNP into BNP-32 ${ }^{[36]}$.

Discovery of corin and furin as the most likely pro-BNP processing enzymes has extended our knowledge of the natriuretic peptide system ${ }^{[37]}$. To date, despite numerous studies devoted to the assessment of the clinical significance of pro-BNP-derived peptides, the clinical importance of corin and furin in the pathophysiology of CVDs remains incompletely understood.

We hypothesized that corin and furin might serve as biomarkers to predict the development of CVDs in T2DM patients. To test this hypothesis, we measured plasma corin and furin in T2DM patients with or without CVDs as well as in healthy subjects. The present study shows that corin levels were significantly lower in T2DM patients with or without CVDs than those in healthy subjects. Similarly, corin levels were lower in T2DM patients with CVDs than those in T2DM patients without CVDs. These results suggest that the reduction of corin levels, may be related more closely to the pathological changes associated with DM than that of CVDs. The reduced levels of corin observed in T2DM patients with CVDs are likely to reflect either the chronic loss of cardiomyocytes and/or diminished cardiac corin protein expression. It is possible that the expression/activity of corin sheddase, which remains unknown, are reduced in CVDs, leading to low levels of plasma corin. Alternatively, corin cleaves itself on the cell surface and in CVDs such a process is inhibited or impaired, resulting in lower corin levels in plasma. Other possibilities may also include accelerated plasma corin degradation or clearance. In the current study, there was a significant negative correlation between corin and BNP levels in T2DM patients with or without CVD. Furthermore, a negative correlation was found between corin and furin levels in T2DM patients with CVD.

It has been previously reported that plasma corin levels were reduced significantly in patients with $\mathrm{HF}$ and that the reduction of corin levels appeared to correlate with the severity of $\mathrm{HF}^{[38,39]}$. These results are consistent with the elevated levels of unprocessed natriuretic peptides in patients with HF, suggesting that corin deficiency may be a contributing factor in failing hearts ${ }^{[40]}$. Recently, low serum corin levels were found to be an independent predictor for poor clinical outcomes in patients with 
coronary disease ${ }^{[41]}$. Interestingly, it has been recently demonstrated that there were no significant differences in the plasma corin concentration/activity among the acute decompensated heart failure (ADHF), non-cardiac dyspnoea (non-ADHF), and CHF patient groups. Additionally, there was no significant correlation between corin concentration/activity and pro-BNP levels, NT-pro-BNP levels, BNP levels, the processing of pro-BNP (NT-pro-BNP/pro-BNP and BNP/pro-BNP), or the degree of pro-BNP glycosylation ${ }^{[42]}$.

Surprisingly, it has been reported that the furin-mediated cleavage of pro-BNP resulted in $\mathrm{BNP}_{1-32}$, whereas corinmediated processing of pro-BNP results in the formation of truncated $\mathrm{BNP}_{4-32}$, suggesting that corin is unlikely to be the primary candidate for the role of pro-BNP processing enzyme ${ }^{[37,43]}$.

In the present study, furin levels appeared to be significantly higher in T2DM patients with CVDs than those in T2DM patients without CVDs and healthy subjects. In addition, furin levels were higher in T2DM patients without CVDs than those in healthy subjects. These results suggest that the elevation of furin levels, may be related more closely to the pathological changes associated with CVDs than that of DM. Furthermore, there was a significant positive correlation between furin and BNP levels in healthy subjects, T2DM patients with or without CVDs. Moreover, a negative correlation was found between furin and corin levels in T2DM patients with CVDs.

Recently, Vodovar et al. reported that plasma furin activity was significantly higher in the patients suffering from ADHF than in patients with non-ADHF and CHF. In addition, there were significant positive correlations between circulating furin activity and plasma levels of pro-BNP, NT-pro-BNP and BNP in the ADHF, nonADHF and CHF groups. Moreover, the activity of circulating furin was found to be negatively correlated with the degree of pro-BNP glycosylation in ADHF and non-ADHF patients but not in CHF patients. Surprisingly, there were no significant differences in the plasma furin concentration among the ADHF, nonADHF and CHF groups. Furthermore, there was no significant correlation between plasma furin concentration and pro-BNP levels, NT-pro-BNP levels, BNP levels, the processing of pro-BNP, or the degree of pro-BNP glycosylation. Contrary to expectations, neither concentrations of furin/corin nor corin activity directly determine the processing of pro-BNP ${ }^{[42]}$.

In our study, the sensitivity of CVDs diagnosis in T2DM patients by furin level was significantly higher than that by corin. On the other hand, both furin and BNP have a similar sensitivity in diagnosing CVDs. Furthermore, the specificity of CVDs diagnosis by furin level was significantly higher than that by BNP or by corin. A recent study by Jin et al. reported that at a cutoff value of $78.2 \mathrm{pg} / \mathrm{ml}$, the BNP level showed a sensitivity of $71.9 \%$ and a specificity of $68.1 \%$ for the diagnosis of PAD in T2DM patients ${ }^{[32]}$. In the current study, since furin produced a similar sensitivity to BNP and better specifi- city, PPV, NPV, diagnostic accuracy and AUC than BNP in the biochemical diagnosis of CVDs, furin measurements could add important information to clinical judgment in establishing a final diagnosis of CVDs.

A substantial body of evidence suggested that genetic determinants, such as the reported corin gene SNPs and others yet to be discovered, may explain some of the variability in the processing of pro-BNP. Our findings demonstrate that the frequency of C1757T or A1796C alleles was not significantly different among the different study groups. Furthermore, corin gene SNPs are not associated with plasma corin levels neither in the study cohort nor in T2DM patients with CVDs. Epidemiological studies of large population-based cohorts have shown that the minor 1757T-1796C corin allele is more common in African-Americans than in Caucasians $(\sim 12 \%$ vs. $<0.2 \%$ carrying one or more copies of the allele) ${ }^{[20]}$. The C1757T and A1796C SNPs result in T555I and Q568P amino acid substitutions, respectively. It has been previously demonstrated that these variants impair the biological activity of corin by impairing its zymogen activation. In functional assays, T555I and Q568P corin variants had slightly lower activities than that of wild-type but the difference was not statistically significant. Moreover, these corin variants had a reduced activity for the processing of natriuretic peptides compared with that of wild type, indicating that these variants may impair corin function, thereby lowering its biological activity ${ }^{[21,44]}$. Despite these considerations, we do not have direct molecular data that the activity of corin is altered as a result of the C1757T and A1796C SNPs, and, therefore, we cannot exclude the possibility that these SNPs may be in linkage disequilibrium with another yet-to-be-identified causal variant. We focused on two corin SNPs that have the highest a priori likelihood of altering protein function. However, it may be argued that a more comprehensive examination of the total allelic variation of the corin locus in more diverse populations using a haplotypebased approach will yield additional insights.

\section{Conclusions}

In conclusion, to the best of our knowledge, the present study is the first to provide a statistical elucidation of the clinical value of the furin levels in the risk assessment of CVDs in T2DM patients. Our findings indicate that a routine measurement of furin levels can improve the predictive ability of CVDs in T2DM patients. Our findings are expected to encourage designing future studies with larger cohorts of patients from different ethnic populations. Further studies are warranted to determine if plasma furin levels are changed in patients with cardiovascular complications over a longer period following medical treatment and if the changes correlate with the underlying pathology. Such studies shall help to understand the diagnostic and prognostic values of furin and may also help to translate basic discoveries in furin research into novel strategies to treat cardiovascular complications. 


\section{Conflict of interest}

The authors declare that they have no conflict of interest.

\section{References}

1) Shaw, J. E., Sicree, R. A. and Zimmet, P. Z. (2010). Global estimates of the prevalence of diabetes for 2010 and 2030. Diabetes Res Clin Pract 87(1): 4-14.

2) Isomaa, B., Almgren, P., Tuomi, T., Forsén, B., Lahti, K., Nissén, M., Taskinen, M. R. and Groop, L. (2001). Cardiovascular morbidity and mortality associated with the metabolic syndrome. Diabetes Care 24(4): 683-689.

3) World Health Organization (WHO) Cardiovascular diseases (CVDs) Fact Sheet, No. 317. March 2013; available at: http://www.who.int/mediacentre/factsheets/fs317/en/.

4) World Health Organization (WHO) Health conditions in the occupied Palestinian territory, including east Jerusalem, and in the occupied Syrian Golan Sixty-fourth World Health Assembly, Provisional agenda item 15, A64/INF.DOC./2. May 2011; available at: http://apps.who.int/gb/ebwha/pdf_files/WHA64/A64_ ID2-en.pdf.

5) McGrath, M. F., de Bold, M. L. and de Bold, A. J. (2005). The endocrine function of the heart. Trends Endocrinol Metab 16(10): 469-477.

6) Potter, L. R., Abbey-Hosch, S. and Dickey, D. M. (2006). Natriuretic peptides, their receptors, and cyclic guanosine monophosphate-dependent signaling functions. Endocr Rev 27(1): 47-72.

7) Munagala, V. K., Burnett, J. C. Jr. and Redfield, M. M. (2004). The natriuretic peptides in cardiovascular medicine. Curr Probl Cardiol 29(12): 707-769.

8) Motiwala, S. R. and Januzzi, J. L. Jr. (2013). The role of natriuretic peptides as biomarkers for guiding the management of chronic heart failure. Clin Pharmacol Ther 93(1): 57-67.

9) Dries, D. L. (2007). Relevance of molecular forms of brain natriuretic peptide for natriuretic peptide research. Hypertension 49(5): 971-973.

10) Ichiki, T., Huntley, B. K., Heublein, D. M., Sandberg, S. M., McKie, P. M., Martin, F. L., Jougasaki, M. and Burnett, J. C. Jr. (2011). Corin is present in the normal human heart, kidney, and blood, with pro-B-type natriuretic peptide processing in the circulation. Clin Chem 57(1): 40-47.

11) Sawada, Y., Suda, M., Yokoyama, H., Kanda T., Sakamaki, T., Tanaka, S., Nagai, R., Abe, S. and Takeuchi, T. (1997). Stretch-induced hypertrophic growth of cardiocytes and processing of brain-type natriuretic peptide are controlled by proproteinprocessing endoprotease furin. J Biol Chem 272(33): 20545-20554.

12) Bugge, T. H., Antalis, T. M. and Wu, Q. (2009). Type II transmembrane serine proteases. J Biol Chem 284(35): 23177-23181.

13) Wu, F., Yan, W., Pan, J., Morser, J. and Wu, Q. (2002). Processing of pro-atrial natriuretic peptide by corin in cardiac myocytes. J Biol Chem 277(19): 16900-16905.

14) Ichiki, T., Huntley, B. K. and Burnett, J. C. Jr. (2013). BNP molecular forms and processing by the cardiac serine protease corin. Adv Clin Chem 61: 1-31.

15) Hooper, J. D., Scarman, A. L., Clarke, B. E., Normyle, J. F. and Antalis, T. M. (2000). Localization of the mosaic transmembrane serine protease corin to heart myocytes. Eur J Biochem 267(23): 6931-6937.

16) Wu, Q., Xu-Cai, Y. O., Chen, S. and Wang, W. (2009). Corin: new insights into the natriuretic peptide system. Kidney Int 75(2): 142-146.

17) Creemers, J. W. and Khatib, A. M. (2008). Knock-out mouse models of proprotein convertases: unique functions or redundancy? Front Biosci 13: 4960-4971.

18) Dries, D. L. (2011). Process matters: Emerging concepts underlying impaired natriuretic peptide system function in heart failure. Circ Heart Fail 4(2):107-110.

19) Ichiki, T., Boerrigter, G., Huntley, B. K., Sangaralingham, S. J., McKie, P. M., Harty, G. J., Harders, G. E. and Burnett, J. C. Jr. (2013). Differential expression of the pro-natriuretic peptide convertases corin and furin in experimental heart failure and atrial fibrosis. Am J Physiol Regul Integr Comp Physiol. 304(2): R102-109.

20) Dries, D. L., Victor, R. G., Rame, J. E., Cooper, R. S., Wu, X., Zhu, X., Leonard, D., Ho, S. I., Wu, Q., Post, W. and Drazner, M. H. (2005). Corin gene minor allele defined by 2 missense mutations is common in blacks and associated with high blood pressure and hypertension. Circulation 112(16): 2403-2410.

21) Wang, W., Liao, X., Fukuda, K., Knappe, S., Wu, F., Dries, D. L., Qin, J. and Wu, Q. (2008). Corin variant associated with hypertension and cardiac hypertrophy exhibits impaired zymogen activation and natriuretic peptide processing activity. Circ Res 103(5): 502-508.

22) American Diabetes Association. (2009). Standards of medical care in diabetes - 2009. Diabetes Care 32(suppl 1): S13-S61.

23) McMurray, J. J., Adamopoulos, S., Anker, S. D., Auricchio, A., Böhm, M., Dickstein, K., Falk, V., Filippatos, G., Fonseca, C., Gomez-Sanchez, M. A., Jaarsma, T., Køber, L., Lip, G. Y., Maggioni, A. P., Parkhomenko, A., Pieske, B. M., Popescu, B. A., Rønnevik, P. K., Rutten, F. H., Schwitter, J., Seferovic, P., Stepinska, J., Trindade, P. T., Voors, A. A., Zannad, F. and Zeiher, A; ESC Committee for Practice Guidelines (2012). ESC Guidelines for the diagnosis and treatment of acute and chronic heart failure 2012: The Task Force for the Diagnosis and Treatment of Acute and Chronic Heart Failure 2012 of the European Society of Cardiology 
Developed in collaboration with the Heart Failure Association (HFA) of the ESC. Eur Heart J. 33(14):1787-1847.

24) Yancy, C. W., Jessup, M., Bozkurt, B., Butler, J., Casey, D. E. Jr., Drazner, M. H., Fonarow, G. C., Geraci, S. A., Horwich, T., Januzzi, J. L., Johnson, M. R., Kasper, E. K., Levy, W. C., Masoudi, F. A., McBride, P. E., McMurray, J. J., Mitchell, J. E., Peterson, P. N., Riegel, B., Sam, F., Stevenson, L. W., Tang, W. H., Tsai, E. J. and Wilkoff, B. L.; American College of Cardiology Foundation; American Heart Association Task Force on Practice Guidelines (2013). 2013 ACCF/AHA guideline for the management of heart failure: a report of the American College of Cardiology Foundation/American Heart Association Task Force on Practice Guidelines. J Am Coll Cardiol 62(16):e147-239.

25) Norusis, M. (2009). SPSS 17.0 advanced statistical procedures companion. Upper Saddle-River, N.J.: Prentice Hall, Inc.

26) Devlin, B. and Risch, N. (1995). A comparison of linkage disequilibrium measures for fine-scale mapping. Genomics 29(2): 311-322.

27) Peakall, R. and Smouse, P. E. (2006). GenAlEx 6: genetic analysis in Excel. Population genetic software for teaching and research. Molecular Ecology Notes 6: 288-295.

28) Peakall, R. and Smouse, P. E. (2012). GenAlEx 6.5: genetic analysis in Excel. Population genetic software for teaching and research-an update. Bioinformatics 28: 2537-2539.

29) McCullough, P. A., Omland, T. and Maisel A. S. (2003). B-type natriuretic peptides: a diagnostic breakthrough for clinicians. Rev Cardiovasc Med 4(2):72-80.

30) Daniels, L. B., Maisel, A. S. (2007). Natriuretic peptides. J Am Coll Cardiol 50(25): 2357-2368.

31) Pfister, R., Sharp, S., Luben, R., Welsh, P., Barroso, I., Salomaa, V., Meirhaeghe, A., Khaw K. T., Sattar, N., Langenberg, C. and Wareham, N. J. (2011). Mendelian randomization study of Btype natriuretic peptide and type 2 diabetes: evidence of causal association from population studies. PLoS Med 8(10):e1001112.

32) Jin, Q. H., Ye, W. L., Chen, H. H., He, X. J., Li, T. L., Liu, Q., Zhong, L., Xu, L. and Han, C. M. (2014). Levels of brain natriuretic peptide are associated with peripheral arterial disease in subjects with type-2 diabetes mellitus. BMC Endocr Disord 14: 27.

33) Wang, T. J., Larson, M. G., Levy, D., Benjamin, E. J., Leip, E. P., Omland, T., Wolf, P. A. and Vasan, R. S. (2004). Plasma natriuretic peptide levels and the risk of cardiovascular events and death. N Engl J Med 350(7): 655-663.

34) Phelan, D., Watson, C., Martos, R., Collier, P., Patle, A., Donnelly, S., Ledwidge, M., Baugh, J. and McDonald, K. (2012). Modest elevation in BNP in asymptomatic hypertensive patients reflects subclinical cardiac remodeling, inflammation and extracellular matrix changes. PLoS One 7(11): e49259.

35) Takahashi, T., Nakamura, M., Onoda, T., Ohsawa M., Tanno, K., Itai, K., Sakata, K., Sakuma, M., Tanaka, F., Makita, S., Yoshida, Y., Ogawa, A., Kawamura, K. and Okayama, A. (2009). Predictive value of plasma B-type natriuretic peptide for ischemic stroke: a community-based longitudinal study. Atherosclerosis 207(1): 298-303.

36) Chen, H. H. (2007). Heart failure: a state of brain natriuretic peptide deficiency or resistance or both! J Am Coll Cardiol 49(10): 1089-1091.

37) Semenov, A. G., Tamm, N. N., Seferian, K. R., Postnikov, A. B., Karpova, N. S., Serebryanaya, D. V., Koshkina, E. V., Krasnoselsky, M. I. and Katrukha, A. G. (2010). Processing of pro-Btype natriuretic peptide: furin and corin as candidate convertases. Clin Chem 56(7):1166-1676.

38) Dong, N., Chen, S., Yang, J., He, L., Liu, P., Zheng, D., Li, L., Zhou, Y., Ruan, C., Plow, E. and $\mathrm{Wu}, \mathbf{Q}$. (2010). Plasma soluble corin in patients with heart failure. Circ Heart Fail 3(2): 207-211.

39) Ibebuogu, U. N., Gladysheva, I. P., Houng, A. K. and Reed, G. L. (2011). Decompensated heart failure is associated with reduced corin levels and decreased cleavage of pro-atrial natriuretic peptide. Circ Heart Fail. 4(2):114-120.

40) Dong, N., Chen, S., Wang, W., Zhou, Y. and Wu, Q. (2012). Corin in clinical laboratory diagnostics. Clin Chim Acta 413(3-4): 378-383.

41) Peleg, A., Ghanim, D., Vered, S. and Hasin Y. (2013). Serum corin is reduced and predicts adverse outcome in non-ST-elevation acute coronary syndrome. Eur Heart J Acute Cardiovasc Care 2(2): 159-165.

42) Vodovar, N., Séronde, M. F., Laribi, S., Gayat, E., Lassus, J., Boukef, R., Nouira, S., Manivet, P., Samuel, J. L., Logeart, D., Ishihara, S., Cohen, Solal, A., Januzzi, J. L. Jr., Richards, A. M., Launay, J. M. and Mebazaa, A; GREAT Network (2014). Post-translational modifications enhance NTproBNP and BNP production in acute decompensated heart failure. Eur Heart J. 35(48): 3434-3441.

43) Semenov, A. G. and Seferian, K. R. (2011). Biochemistry of the human B-type natriuretic peptide precursor and molecular aspects of its processing. Clin Chim Acta 412(11-12):850-860.

44) Rame, J. E., Tam, S. W., McNamara, D., Worcel, M., Sabolinski, M. L., Wu, A. H. and Dries, D. L. (2009). Dysfunctional corin i555(p568) allele is associated with impaired brain natriuretic peptide processing and adverse outcomes in blacks with systolic heart failure: results from the Genetic Risk Assessment in Heart Failure substudy. Circ Heart Fail 2(6):541-548. 